\title{
El neutrófilo y su importancia en la enfermedad periodontal Artículo de Revisión
}

\author{
Díaz-Alfaro L*, González-Facio P**
}

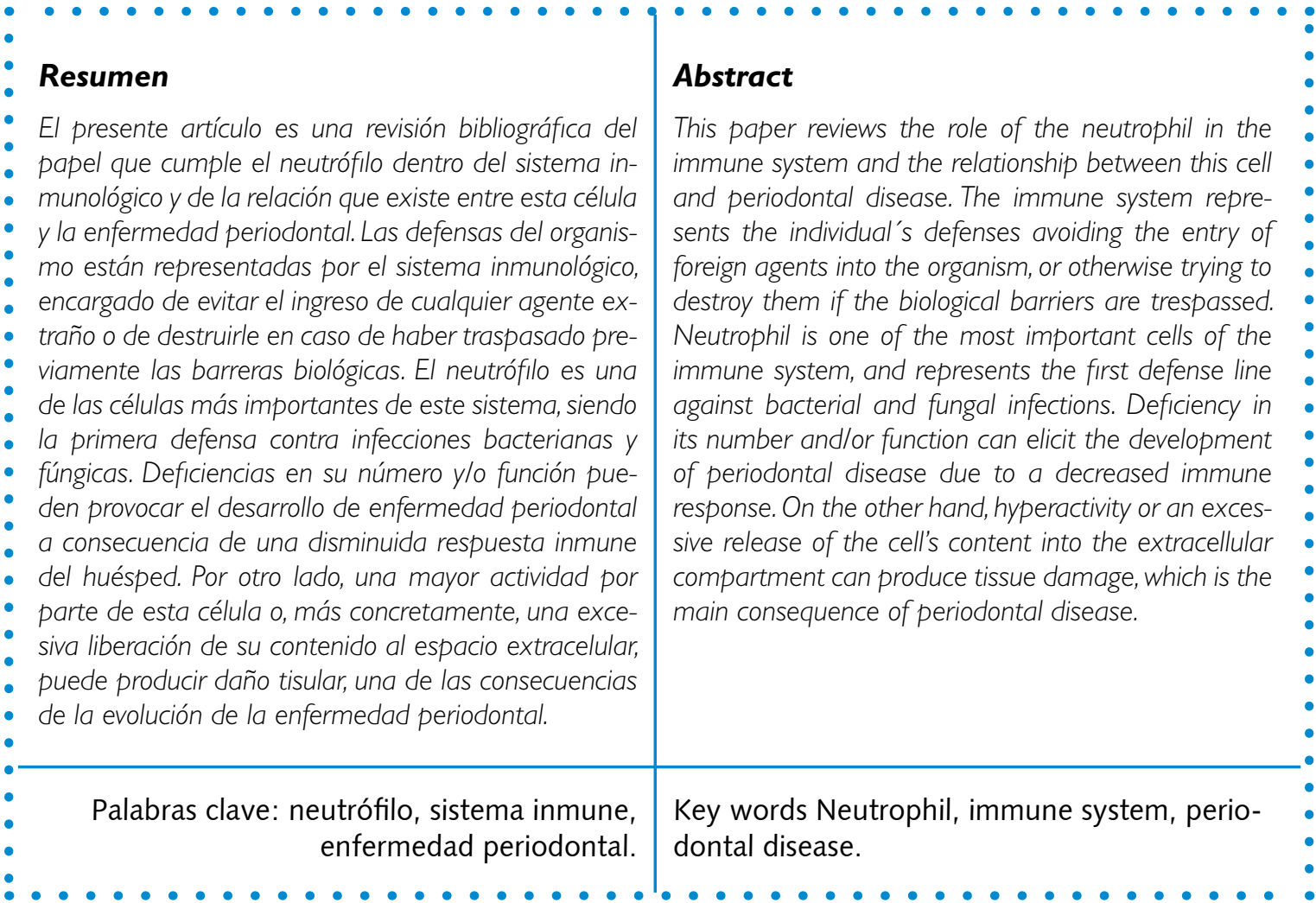

\section{Introducción}

El cuerpo humano tiene la capacidad de ser resistente a una enorme variedad de microorganismos y tóxicos capaces de lesionar los tejidos. Esta capacidad se denomina inmunidad.

El sistema inmune es un complejo entramado de células, y diferentes moléculas y receptores encargados de evitar el ingreso de 
agentes extraños al organismo o, en su defecto, de evitar el daño que pueden producir estos antígenos en caso de traspasar las primeras barreras e introducirse en el organismo.

Una gran parte de la inmunidad en el ser humano es adquirida después de que el cuerpo sea atacado por un microorganismo o toxina, y necesita a menudo semanas o meses para desarrollarse. Otra parte de la inmunidad se desarrolla de forma innata, y es el resultado de procesos generales inespecíficos, en lugar de procesos dirigidos contra agentes específicos. Por tanto, de forma general, el sistema inmune se puede dividir en dos grandes grupos: Inmunidad natural o congénita e Inmunidad específica o adquirida.

Dentro de la inmunidad congénita podríamos hablar de una primera línea de defensa formada por barreras mecánicas (ej. piel y mucosas), barreras físicas (ej. tos, estornudos), barreras químicas (ej. secreciones sebáceas, acidez gástrica) y barreras biológicas (ej. microorganismos comensales). Si estas barreras no fueron suficientemente eficaces para evitar el ingreso del agente extraño en el organismo, será la segunda línea de defensa (perteneciente también a la inmunidad congénita) la encargada de producir su destrucción. A este grupo pertenecen los neutrófilos, monocitos, macrófagos, células natural killer, sistema del complemento y sustancias humorales inespecíficas, entre otras.

Por último, la inmunidad específica o adquirida se corresponde con la última línea de defensa y se divide a su vez en dos tipos: Inmunidad humoral (mediada por anticuerpos) e Inmunidad celular (mediada por linfocitos).

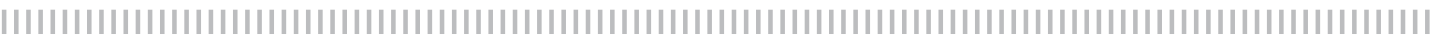

\section{Enfermedad periodontal}

La periodontitis es una enfermedad infecciosa que induce una respuesta inflamatoria de la encía y de los tejidos de soporte de los dientes, causando pérdida de inserción epitelial y ósea. El desarrollo de la enfermedad depende de la susceptibilidad del huésped y la presencia de una flora microbiana patógena estando involucradas bacterias gram-negativas.

Las enfermedades periodontales, son la gingivitis que es un proceso inflamatorio limitado a la encía, y la periodontitis que es una condición infecciosa que da como resultado la destrucción del ligamento periodontal y del hueso alveolar.

La destrucción periodontal es el resultado de la acción de varios productos tóxicos que liberan patógenos específicos de la placa bacteriana, también como la respuesta del huésped en contra de la placa bacteriana y sus productos. Se sabe que la respuesta del huésped a infecciones periodontales da como resultado la producción local exagerada de citoquinas y mediadores biológicos incluyendo interleucinas y prostaglandinas que pueden exacerbar algunas de las enfermedades sistémicos.

\section{Función del neutrófilo}

Los neutrófilos ó leucocitos polimorfonucleares (PMN) son componentes esenciales del sistema inmune natural. Son las principales células fagocíticas encontradas en sangre periférica; correspondiéndose con un 50$70 \%$ del total de células de la serie blanca ${ }^{1}$.

Se les considera la primera línea de defensa contra infecciones bacterianas y fún- 
gicas (además de las barreras naturales anteriormente citadas).

Son producidos en la médula ósea a partir de células madre mieloides, por medio del proceso denominado "fagocitopoyesis". Posteriormente, los neutrófilos circulan en sangre durante períodos relativamente cortos ya que su vida media es de sólo 8-20 horas en circulación, aumentando varias veces esta vida media al entrar en tejidos infectados o inflamados ${ }^{2}$.

Dentro de las células defensivas, los neutrófilos son los primeros en llegar al tejido afectado, seguidos por los macrófagos y linfocitos. La lesión periodontal inicial aparece a los cuatro días de acumulación de placa. No se evidencia clínicamente pero se caracteriza por un aumento del líquido gingival crevicular (LGC) y migración de neutrófilos desde el plexo vascular hacia el epitelio de inserción y surco gingival ${ }^{3}$.

Este desplazamiento del neutrófilo desde los capilares sanguíneos hasta la lesión consta de varias etapas: (Fig. 1)
1 Marginación: contacto de los PMN con las paredes endoteliales a causa de la estasis vascular.

2 Adherencia al endotelio: interacción entre glucoproteínas superficiales de los neutrófilos y su correspondiente receptor en células endoteliales (selectina $\mathrm{E}$ ). También se produce interacción entre integrina 2 (presente también en otros leucocitos, no sólo en PMN) e ICAM1 (molécula de adhesión intercelular 1) presente en células endoteliales.

3 Diapédesis: es la migración transendotelial. Se activan proteínas contráctiles (actina y miosina) y la célula fagocítica emite pseudópodos que se introducen entre las uniones de las células endoteliales. Posteriormente irán atravesando el endotelio y la membrana basal hasta llegar al espacio extravascular. Para que esto suceda es necesario que la célula sea atraída hacia el foco de infección. Este fenómeno se denomina "quimiotaxis" y es llevado a cabo por medio de

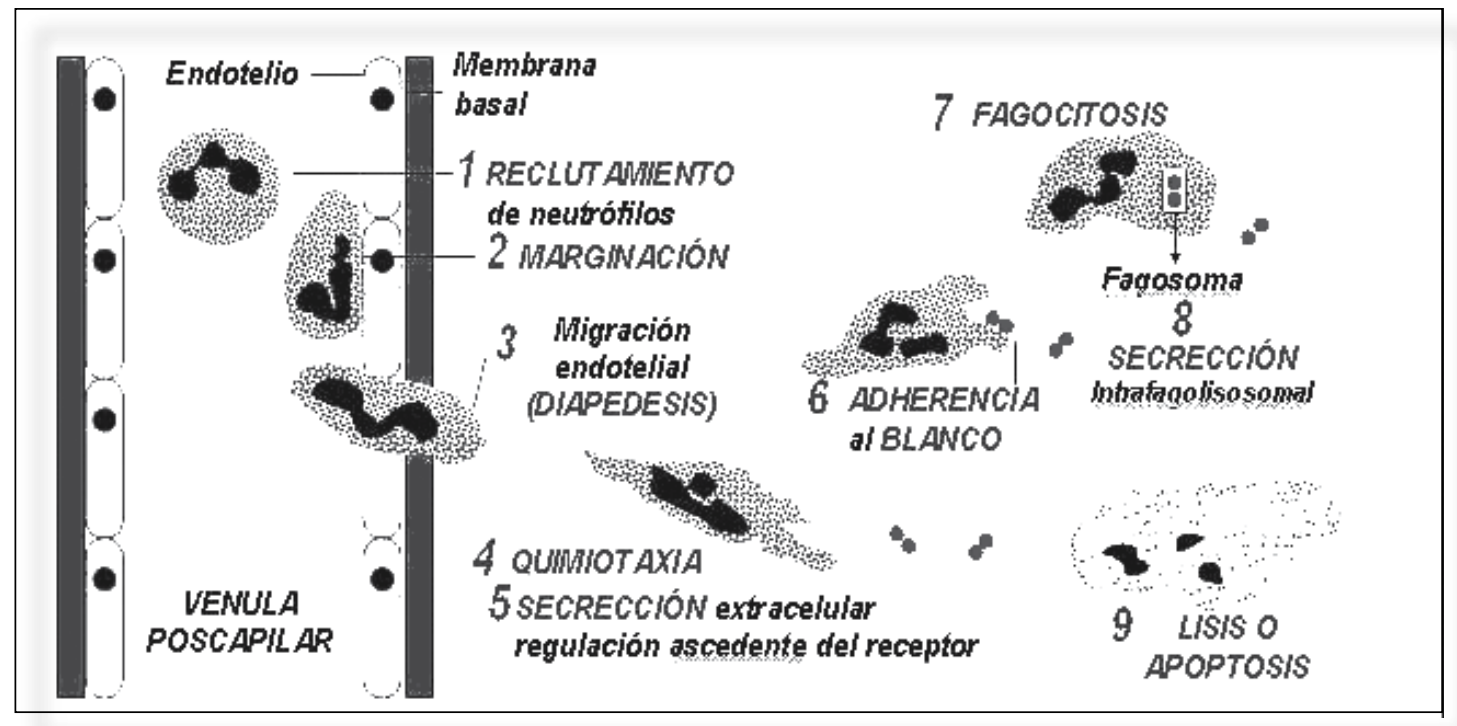

Fig. 1. Esquema del Proceso de diapédesis y fagocitosis del Netrófilo (Miyasaki) 
diferentes moléculas como: interleucina 8 (IL-8), factor C5a del complemento (C5a), leucotrieno B4 (LTB4), péptidos de $\mathrm{N}$-formil y factores de activación plaquetaria.

4 Fagocitosis y muerte celular: al llegar los PMN al lugar de la lesión, éstos reconocen moléculas específicas en la superficie del invasor para, posteriormente, invaginarlo y destruirlo. Este proceso es facilitado por medio de proteínas opsónicas, como por ejemplo inmunoglobulinas o fragmento $\mathrm{C} 3 \mathrm{~b}$ del complemento.

La destrucción del microorganismo en el interior del neutrófilo se produce a través de dos mecanismos diferentes:

1 Mecanismo óxido-dependiente: La oxidasa NADPH de la membrana de los fagolisosomas reduce el oxígeno (O2) a superóxido (O.). A continuación, se formará peróxido de hidrógeno que, en com-binación con mieloperoxidasa, dará lugar a la formación de ácido hipocloroso, potente microbicida. Todos estos compuestos dependientes del $\mathrm{O} 2$ tienen gran potencial tóxico.

2 Mecanismo óxido-independiente Agentes antimicrobianos, no dependientes del O2, ejercen su acción en el fagolisosoma durante la fagocitosis.

Entre los diferentes agentes antimicrobianos, destacan la elastasa y metaloproteinasas que, además de su actividad antibacteriana, tienen potencial para destruir tejidos extracelulares.

Una vez que el neutrófilo ha cumplido su función, muere por apoptosis (muerte celular programada) y es eliminado por macrófagos para evitar la posible liberación de su contenido citotóxico al medio extracelular, hecho que podría ocurrir en caso de muerte por necrosis.

\section{Alteración en neutrófilos}

La literatura referente al papel del neutrófilo en la enfermedad periodontal contempla diferentes condiciones que determinan la relación que existe entre esta célula y la enfermedad periodontal. Por un lado, algunos autores hablan de "hipofunción" del neutrófilo, otros de "hiperfunción" y, por último, otros prefieren utilizar un término más general; "disfunción". Nosotros nos decantamos por esta última terminología ya que engloba todas las posibilidades.

La "hipofunción" neutrofílica se refiere a aquellos casos en los que existe una disminución de la función de los neutrófilos, ya sea por disminución de su número total (Síndrome de deficiencia de adhesión leucocitaria, Síndrome de Wiskott Aldrich), o por ausencia o disminución de la destrucción intracelular tras la fagocitosis (enfermedad granulomatosa crónica, deficiencia de mieloperoxidasa).

Además, numerosos autores sugieren que algunos periodontopatógenos poseen factores de virulencia que pueden destruir los neutrófilos o disminuir su función4. La leucotoxina producida por Actinobacillus actinomiycetemcomitans (A.a) ${ }^{5-7}$ y proteasas producidas por Porphyromonas gingivalis $(\mathrm{Pg})^{8,9}$ son algunos ejemplos.

El término "hiperfunción" hace referencia al exceso de actividad y liberación de productos tóxicos por parte del neutrófilo; responsable, en parte, de la destrucción tisular en la Enfermedad Periodontal ${ }^{10}$.

Se han observado alteraciones en la quimiotaxis en algunas formas de enfermedad periodontal, fundamentalmente en periodontitis agresivas localizadas (PAL). Existen diferentes causas, entre las que encontramos: disminución de IL-811; disminución de $\mathrm{C} 5 \mathrm{a}^{11,12}$; disminución de fMLP (péptidos de $\mathrm{N}$-formilmetionil) ${ }^{12-14}$; y disminución de la glicoproteína superficial GP110 ${ }^{15}$, aunque todavía existen dudas sobre su influencia.

Los defectos quimiotácticos en pacientes con periodontitis agresivas están presentes tanto en células aisladas de sangre periférica como en el lugar de la lesión, y persisten incluso tras la eliminación de los patógenos y resolución de la lesión inflamatoria ${ }^{11}$. Se considera que estos defectos se heredan de forma autosómica dominante ${ }^{16}$. 
En esta forma de EP deben además tenerse en consideración hallazgos como el de Wilson y Hamilton que observaron una respuesta a Aa mediada predominantemente por IgG2 en pacientes con PAL. Al ser una inmunoglobulina con débil capacidad opsonizante, estos autores propusieron esta elevada cantidad de IgG2 como un marcador de riesgo.

Otras causas que explican la disminución de la quimiotaxis en PAL son la disminución de calcio intracelular ${ }^{10,17,18,}$ disminución de la actividad de la protein-cinasa C19, y disminución de la actividad de la diacilglicerol cinasa ${ }^{20}$.

Otro tipo de alteraciones de los neutrófilos son las relativas a la fagocitosis de pacientes con $\mathrm{PAL}^{21-23}$ y con periodontitis crónica ${ }^{24-}$ ${ }^{26}$, descritas en diversos trabajos.

El tabaco es otro factor que influye en la respuesta de los neutrófilos. Mariggió y $\mathrm{cols}^{28}$ observaron un aumento en la apoptosis de los neutrófilos obtenidos del FGC de pacientes fumadores con periodontitis crónica. Por tanto, el tabaco se consideraría causa de una deficiente respuesta del huésped. Otro estudio ${ }^{29}$ encontró un aumento en la producción de $\mathrm{H}^{2} \mathrm{O}^{2}$ durante el estallido oxidativo de los neutrófilos expuestos a tabaco in vitro. A su vez, en otro trabajo ${ }^{30}$ se observó una mayor fagocitosis por parte de PMN en saliva al estar expuestos a tabaco.

\section{Daño tisular}

En la inflamación aguda, el número de neutrófilos en el tejido puede ser extremadamente alto por la gran llegada desde el torrente sanguíneo y por la disminución en su apoptosis debido a la acción de mediadores inflamatorios locales ${ }^{31}$. Así, la capacidad de daño tisular, por medio de la liberación de radicales de oxígeno y enzimas proteolíticas, será muy alta. Por tanto, la apoptosis juega un papel fundamental en el desarrollo de esta enfermedad.

Los radicales de oxígeno (superóxido, peróxido de hidrógeno y ácido hipocloroso) producidos por los PMN pueden dañar moléculas muy importantes, como proteínas, lípidos, carbohidratos y ácidos nucleicos. La producción de $\mathrm{O}_{2}$ es un paso crucial para el desarrollo de la actividad citotóxica ya que, a partir de éste, se forma el anión superóxido $\left(\mathrm{O}_{2}{ }^{\circ}\right)$ por medio de la oxidasa NADPH. Posteriormente se formará el peróxido de hidrógeno que, gracias a la mieloperoxidasa, dará lugar al ácido hipocloroso, de gran actividad citotóxica, fundamentalmente debido a la producción de cloraminas oxidativas ${ }^{32}$.

En la degranulación de los PMN también se liberan enzimas proteolíticas. La elastasa puede hidrolizar diferentes proteínas de la matriz extracelular como elastina, fibronectina y colágeno tipo III y IV. La colagenasa también es capaz de degradar el colágeno tisular.

Deguchi y cols ${ }^{33}$ fueron los primeros en demostrar el daño periodontal producido por los PMN. Observaron el aumento de la adherencia de PMN a fibroblastos del ligamento periodontal al ser estimulados por lipopolisacáridos (LPS).

Como ya se ha comentado, la apoptosis juega un papel fundamental en la producción de daño tisular. Existen diferentes moléculas inflamatorias que retrasan esta apoptosis, dando lugar a un aumento de PMN en el lugar de la lesión, aumentando las posibilidades de destrucción de los mismos y liberación de sus productos tóxicos al espacio extracelular con el consiguiente daño tisular. La apoptosis de neutrófilos es un importante mecanismo que mantiene un número apropiado de células bajo condiciones fisiológicas. La disminución de este mecanismo se ha asociado con la aparición de diferentes enfermedades inflamatorias, tanto agudas como crónicas y parece estar mediado en gran medida por la excesiva producción de factor estimulador de colonias de granulocitos (G-CSF) y factor estimulador de colonias de granulocitos y macrófagos (GM-CSF) ${ }^{34,35}$. Otros estudios han demostrado la influencia de LPS, C5a, Fmlp $^{36}$, ATP $^{37}$, LTB4 ${ }^{38}$, IL-1 ${ }^{39}$, IL-2 ${ }^{40}$, IL$6^{41}, \mathrm{IL}-15^{42}, \mathrm{IFN}-{ }^{43-44}$ en el retraso de la apoptosis. 


\section{Conclusiones}

Los neutrófilos pueden influir de dos formas diferentes en el desarrollo de la periodontitis. Por un lado pueden estar disminuidos en número y/o función por lo que se produciría una respuesta defensiva deficiente por parte del huésped. Esta posibilidad es más frecuente en aquellas periodontitis con algún trastorno sistémico. Por otro lado, el neutrófilo puede participar en el desarrollo de la enfermedad periodontal al producir daño tisular por medio de sus radicales de oxígeno y enzimas proteolíticas; hecho favorecido por un retraso en la apoptosis.

La enfermedad periodontal es un proceso multifactorial en el que resulta muy complicado describir con exactitud el papel que cumplen cada una de las células y moléculas que participan en el mismo. Se necesita un gran esfuerzo por parte de la comunidad científica para conocer con más detalle el complejo entramado inmunológico que se produce alrededor de esta enfermedad.

\section{Bibliografía}

1 Lakshman R, Finn A. Neutrophil disorders and their management. J Clin Pathol. 2001 Jan; 54(1):7-19.

2 Edwards SW. Biochemistry and physiology of the neutrophil. Cambridge University Press 1994, New York.

3 Kinane DF. Causas y patogenia de la enfermedad periodontal. Periodontology 2000 (edición española). 2002; 1: 8-20.

4 Potempa J, Banbula A, Travis J. Role of bacterial proteinases in matrix destruction and modulation of host responses. Periodontol 2000. 2000 Oct; 24:153-92.

5 Gangbar S, Overall CM, McCulloch CA, Sodek J. Identification of polymorphonuclear leukocyte collagenase and gelatinase activities in mouthrinse samples: correlation with periodontal disease activity in adult and juvenile periodontitis. J Periodontal Res. 1990 Sep; 25(5):257-67.

6 Haraszthy VI, Hariharan G, Tinoco EM, Cortelli JR, Lally ET, Davis E, Zambon JJ. Evidence for the role of highly leukotoxic Actinobacillus actinomycetemcomitans in thepathogenesis of localized juvenile and other forms of early-onset periodontitis. J Periodontol. 2000 Jun; 71(6):912-22.

7 Schenkein HA. Etiology of localized juvenile periodontitis. J Periodontol 1998; 69: 1068-9.

8 Curtis MA, Aduse-Opoku J, Rangarajan M. Cysteine proteases of Porphyromonas gingivalis. Crit Rev Oral Biol Med. 2001; 12(3):192-216

9 Kadowaki T, Nakayama K, Okamoto K, Abe N, Baba A, Shi Y, Ratnayake DB, Yamamoto K. Porphyromonas gingivalis proteinases as virulence determinants in progression of periodontal diseases. J Biochem 2000 Aug; 128(2):153-9.
10 Kantarci A, Oyaizu K, Van Dyke TE. Neutrophil-mediated tissue injury in periodontal disease pathogenesis: findings from localized aggressive periodontitis. J Periodontol. 2003 Jan; 74(1):66-75.

11 Van Dyke TE. Role of the neutrophil in oral disease: receptor deficiency in leukocytes from patients with juvenile periodontitis. Rev Infect Dis. 1985 MayJun; $7(3): 419-25$.

12 Vn Dyke TE, Warbington $M$, Gardner $M$, Offenbacher S. Neutrophil surface protein markers as indicators of defective chemotaxis in LJP. J Periodontol. 1990 Mar; 61(3):180-4

13 De Nardin E. The role of inflammatory and immunological mediators in periodontitis and cardiovascular disease. Ann Periodontol. 2001 Dec; 6(1):30-40.

14 De Nardin E, De Luca C, Levine MJ, Genco RJ. Antibodies directed to the chemotactic factor receptor detect differences between chemotactically normal and defective neutrophils from LJP patients. J Periodontol 1990; 61: 609-617.

15 Van Dyke TE, Hoop GA. Neutrophil function and oral disease. Crit Rev Oral Biol Med. 1990; 1(2):117-33.

16 Hart TC, Marazita ML, Schenkein HA, Diehl SR. Reinterpretation of the evidence for $\mathrm{X}$-linked dominant inheritance of juvenile periodontitis. J Periodontol. 1992 Mar; 63(3):169-73. J Periodontol. 1992 Mar; 63(3):169-73.

17 Agarwal S, Reynolds MA, Duckett LD, Suzuki JB. Altered free cytosolic calcium changes and neutrophil chemotaxis in patients with juvenile periodontitis. J

Periodontal Res. 1989 Mar; 24(2):149-54.

18 Daniel MA, McDonald G, Offenbacher S, Van Dyke TE. Defective chemotaxis and calcium response in localized juvenile periodontitis neutrophils. J Periodontol. 1993 Jul; 64(7):617-21. 
19 Kurihara $\mathrm{H}$, Murayama $\mathrm{Y}$, Warbington $\mathrm{ML}$, Champagne CM, Van Dyke TE. Calcium-dependent protein kinase $C$ activity of neutrophils in localized juvenile periodontitis. Infect Immun. 1993 Aug; 61(8):3137-42.

20 Tyagi SR, Uhlinger DJ, Lambeth JD, Champagne C, Van Dyke TE. Altered diacylglycerol level and metabolism in neutrophils from patients with localized juvenile periodontitis. Infect Immun. 1992 Jun; 60(6):2481-7.

21 Cianciola LJ, Park BH, Bruck E, Mosovich L, Genco RJ. Prevalence of periodontal disease in insulin-dependent diabetes mellitus (juvenile diabetes). J Am Dent Assoc. 1982 May; 104(5):653-60.

22 Kalmar JR, Arnold RR, van Dyke TE. Direct interaction of Actinobacillus actinomycetemcomitans with normal and defective (LJP) neutrophils. J Periodontal Res. 1987 May; 22(3):179-81.

23 Wilson ME, Genco RJ, Snyderman R.The phagocytic cell: summary. Rev Infect Dis. 1985 May-Jun; 7(3):387-9.

24 Colombo AP, Eftimiadi C, Haffajee AD, Cugini MA, Socransky SS. Serum IgG2 level, Gm(23) allotype and FcgammaRlla and FcgammaRIIlb receptors in refractory periodontal disease. J Clin Periodontol. 1998 Jun; 25(6):465-74.

25 Miyazaki A, Kobayashi T, Suzuki T, Yoshie H, Hara K. Loss of Fc gamma receptor and impaired phagocytosis of polymorphonuclear leukocytes in gingival crevicular fluid. J Periodontal Res. 1997 Jul; 32(5):43946.

26 Sugita N, Suzuki T, Yoshie H,Yoshida N, Adachi M, Hara K. Differential expression of CR3, Fc epsilon RII and Fc gamma RIII on polymorphonuclear leukocytes in gingival crevicular fluid. J Periodontal Res. 1993 Sep;28(5):363-72.

27 Wilson $M E$, Hamilton RG. Immunoglobulin G subclass response of localized juvenile periodontitis patients to Actinobacillus actinomycetemcomitans Y4 lipopolysaccharide. Infect Immun. 1992 May; 60(5):1806-12.

28 Mariggio MA, Guida L, Laforgia A, Santacroce R, Curci E,Montemurro P, Fumarulo R. Nicotine effects on polymorphonuclear cell apoptosis and lipopolysaccharideinduced monocyte functions. A possible role in periodontal disease? J Periodontal Res. 2001 Feb; 36(1):32-9

29 Ryder MI, Fujitaki R, Johnson G, Hyun W. Alterations of neutrophil oxidative burst by in vitro smoke exposure: implications for oral and systemic diseases. Ann Periodontol. 1998 Jul; 3(1):76-87.

30 Numabe $Y$, Ogawa T, Kamoi H, Kiyonobu K, Sato S, Kamoi K, Deguchi S. Phagocytic function of salivary PMN after smoking or secondary smoking. Ann Periodontol. 1998 Jul; 3(1):102-7.

31 Akgul C, Moulding DA, Edwards SW. Molecular control of neutrophil apoptosis. FEBS Lett. 2001 Jan 5; 487(3):318-22.

32 Waterman WH, Sha'afi RI. A mitogen-activated protein kinase independent pathway involved in the phosphorylation and activation of cytosolic phos- pholipase A2 in human neutrophils stimulated with tumor necrosis factor- alpha. Biochem Biophys Res Commun. 1995 Apr 6; 209(1):271-8.

33 McDonald PP, Bald A, Cassatella MA. Activation of the NFkappaB pathway by inflammatory stimuli in human neutrophils. Blood. 1997 May 1; 89(9):3421-33.

34 Dibbert $B$, Weber $M$, Nikolaizik WH, Vogt $P$, Schoni $\mathrm{MH}$, Blaser K, Simon HU. Cytokine-mediated Bax deficiency and consequent delayed neutrophil apoptosis: a general mechanism to accumulate effector cells in inflammation. Proc Natl Acad Sci U S A. 1999 Nov 9; 96(23):13330-5.

35 Saba S, Soong G, Grenberg S, Prince A. Bacterial stimulation of epithelial G-CSF and GM-CSF expression promotes PMN survival in CF airways. Am J Respir Cell Mol Biol 2002; 27: 561-7.

36 Lee A,Whyte MK, Haslett C. Inhibition of apoptosis and prolongation of neutrophil functional longevity by inflammatory mediators. J Leukoc Biol. 1993 Oct; 54(4):283-8

37 Gasmi L, McLennan AG, Edwards SW. The diadenosine polyphosphates Ap3A and Ap4A and adenosine triphosphate interact with granulocyte-macrophage colonystimulating factor to delay neutrophil apoptosis: implications for neutrophil: platelet interactions during inflammation. Blood. $1996 \mathrm{Apr} 15$; 87(8):3442-9

38 Lee $E$, Lindo $T$, Jackson $N$, Meng-Choong L, Reynolds $P$, Hill $A$, Haswell $M$, Jackson $S$, Kilfeather $S$. Reversal of human neutrophil survival by leukotriene $\mathrm{B}(4)$ receptor blockade and 5-lipoxygenase and 5-lipoxygenase activating protein inhibitors. Am J Respir Crit Care Med. 1999 Dec; 160(6):2079-85.

39 Colotta F, Re F, Polentarutti N, Sozzani S, Mantovani A. Modulation of granulocyte survival and programmed cell death by cytokines and bacterial products. Blood. 1992 Oct 15; 80(8):2012-20.

40 Pericle F, Liu JH, Diaz JI, Blanchard DK, Wei S, Forni G, Djeu JY. Interleukin-2 prevention of apoptosis in human neutrophils. Eur J Immunol. 1994 Feb; 24(2):440-4.

41 Biffl WL, Moore EE, Moore FA, Barnett CC Jr. Interleukin-6 suppression of neutrophil apoptosis is neutrophil concentration dependent. J Leukoc Biol. 1995 Nov; 58(5):582-4.

42 Girard D, Paquet ME, Paquin R, Beaulieu AD. Differential effects of interleukin-15 (IL-15) and IL-2 on human neutrophils: modulation of phagocytosis, cytoskeleton rearrangement, gene expression, and apoptosis by IL-15. Blood. 1996 Oct 15; 88(8):317684.

43 Klebanoff SJ, Olszowski S, Van Voorhis WC, Ledbetter JA, Waltersdorph AM, Schlechte KG. Effects of gamma-interferon on human neutrophils: protection from deterioration on storage. Blood. 1992 Jul 1; 80(1):225-34

44 Daigle I, Yousefi S, Colonna M, Green DR, Simon HU. Death receptors bind SHP-1 and block cytokineinduced anti-apoptotic signaling in neutrophils. Nat Med. 2002 Jan; 8(1):61-7. 